\title{
miR-155-5p regulates macrophage $M 1$ polarization and apoptosis in the synovial fluid of patients with knee osteoarthritis
}

\author{
GUI-SHI LI $^{1 *}$, LEI CUI $^{2 *}$ and GUANG-DA WANG ${ }^{1}$ \\ Departments of ${ }^{1}$ Joint Orthopaedics and ${ }^{2}$ Opthalmology Surgery, \\ Yantai Yuhuangding Hospital, Yantai, Shandong 264000, P.R. China
}

Received July 7, 2019; Accepted April 6, 2020

DOI: $10.3892 /$ etm.2020.9500

\begin{abstract}
Osteoarthritis (OA) is one of the most prevalent degenerative joint diseases that affects millions of individuals worldwide. During OA, proinflammatory factors (including IL-1, IL-6, IL-17 and TNF- $\alpha$ ) are released from chondrocytes and proliferating synoviocytes potentiate the proinflammatory microenvironment of the synovial fluid (SF). The altered SF microenvironment affects the infiltration, polarization and apoptosis of macrophages, though the underlying mechanisms are not completely understood. In the present study, the hypothesis that the knee synovial fluid of patients with knee osteoarthritis (KOA SF) promotes the polarization of peripheral blood mononuclear cell (PBMC)-derived M1 macrophages and inhibits PBMC-derived macrophage apoptosis was investigated. KOA SF increased PBMC-derived macrophage M1 polarization via the microRNA (miR)-155-5p/suppressor of cytokine signaling 1 signaling pathway. Caspase-3 (CASP3) was identified as a novel target of miR-155-5p, where KOA SF inhibited macrophage apoptosis via the miR-155-5p/CASP3 signaling pathway. The results suggested that the proinflammatory environment of KOA SF promoted macrophage M1 polarization and reduced macrophage apoptosis via miR-155-5p. The results provided a potential explanation for the increased number of M1 macrophages observed in KOA SF during OA. In addition, the present study suggested that miR-155-5p may serve as a potential therapeutic target for KOA.
\end{abstract}

\section{Introduction}

Osteoarthritis (OA) is one of the most common degenerative joint diseases, affecting millions of individuals worldwide. OA is characterized by chondrocyte apoptosis and hypertrophy $(1,2)$,

Correspondence to: Professor Guang-Da Wang, Department of Joint Orthopaedics, Yantai Yuhuangding Hospital, 20 Yuhuangding East Road, Zhifu, Yantai, Shandong 264000, P.R. China

E-mail: ydyk2016@126.com

"Contributed equally

Key words: knee osteoarthritis, synovial fluid, microRNA-155-5p, macrophages, apoptosis formation of osteophytes and inflammation of the synovial membrane (3). An increasing number of studies have reported that chronic inflammation with mild synovitis, elevated proinflammatory cytokine levels and infiltration of inflammatory cells (4) are linked to the progression of OA (5). During OA, cartilage integrity is lost and hypertrophic chondrocytes exhibit increased synthetic activity in an attempt to repair the damage. However, as a result of the increased synthetic activity, proinflammatory mediator products [including tumor necrosis factor (TNF)- $\alpha$ to interleukin (IL)-6 and IL-1 $\beta$ )] deregulate chondrocyte function and act on the adjacent synovium to stimulate proliferative and inflammatory responses (6). Proliferating synoviocytes also release proinflammatory products into the synovial fluid (SF), leading to a proinflammatory microenvironment (6). The altered SF microenvironment affects inflammatory cell infiltration, polarization and apoptosis. In addition, various proinflammatory factors such as IL-1, IL-1 $\beta$, IL- 6 and TNF- $\alpha$ secreted by the increased number of inflammatory cells, including M1 macrophages, promote the proinflammatory microenvironment of SF further to exacerbate OA. Previous studies have reported that mononuclear cells, including macrophages (7), B (8) and T cells (9), which aggregate during OA synovial tissues and fluid (4,6-8), contribute to chondrocyte degradation, local inflammation and activation of innate immune cells. A number of studies have demonstrated that the elevated number of macrophages present in the inflamed synovium and SF serve a major role during OA progression (10-12). Therefore, identifying the potential mechanism underlying the effects of the SF microenvironment on macrophage infiltration, apoptosis and polarization is important for identifying the mechanism underlying OA.

Macrophages serve important roles in innate immunity, displaying a high degree of plasticity and polarize into two main subtypes: Classically activated M1 macrophages and alternatively activated M2 macrophages $(13,14)$. Previous studies have reported that M1 macrophages can be activated by interferon- $\gamma$ and toll-like receptors, which display a proinflammatory phenotype by producing proinflammatory cytokines, including IL-1, IL-6, TNF- $\alpha$ and IL-12 $(15,16)$. Macrophages can also be polarized into an anti-inflammatory phenotype, known as M2 macrophages, which promote $\mathrm{T}$ helper cell 2 responses and contribute to tissue repair and healing $(17,18)$. During OA, macrophages can be identified 
by measuring the expression of their respective cell markers using flow cytometry (FCM), including CD14, CD163 and CD68 (19). A previous study reported that local inflammation and activation of innate immune cells, primarily M1 macrophages, are major factors that contribute to the acceleration of OA progression (20). However, few studies have explored the impact of the proinflammatory microenvironment, including synovial tissues and SF, on macrophage infiltration, apoptosis and polarization during OA progression.

MicroRNAs (miRNAs/miRs) are small, single-stranded RNAs that are associated with a number of different diseases, including OA, diabetes and cancer (21-23). miRNAs control the differentiation and function of myeloid and lymphoid cells, among other cell types (24). A recent study on miRNAs in the immune system demonstrated that miR-146a and miR-155-5p are associated with OA (25). Additionally, it has been reported that increasing the expression of miR-155-5p leads to M1 polarization in the RAW264.7 macrophage cell line $(26,27)$. It has also been reported that miR-155-5p promoted M1 macrophage polarization by targeting suppressor of cytokine signaling 1 (SOCS1), which is involved in STAT3 and AKT signaling (28-30). In the present study, the impact of knee SF from patients with knee OA (KOA) on macrophage polarization and apoptosis was investigated. miR-155-5p was identified as a potential target that affected macrophage apoptosis and polarization in KOA SF.

\section{Materials and methods}

Collection of clinical samples. A total of 53 SF samples were collected by needle aspiration from the knee joints of patients with KOA (28 males and 25 females; age range, 50-79 years; average age, 66.7 years; admitted from January 2017 to December 2018; last follow up occurred 1 year after admission) receiving treatment at the Yantai Yuhuangding Hospital (Yantai, China). The present study was approved by the Ethics Committee of Yantai Yuhuangding Hospital and written informed consent was obtained from all participants. Blood samples $(20 \mathrm{ml})$ from healthy subjects (20 males, 22 females; age range, 50-79 years; average age, 68.1 years; admitted from January 2017 to December 2018; last follow up occurred 1 year after admission) and patients with KOA were collected into sodium heparin Vacutainer ${ }^{\circledR}$ tubes (cat. no. 366480; Becton-Dickinson and Company). The severity of radiographic X-ray KOA was graded using the Kellgren/Lawrence scoring system (24), which is scored from 0 to 4 . The X-ray presentations of the patients were as follows: i) Stage $2 \mathrm{KOA}, 9$ patients; ii) stage $3 \mathrm{KOA}$, 28 patients; and iii) stage $3 \mathrm{KOA}, 15$ patients. KOA SF was carefully cross-examined and processed within $2 \mathrm{~h}$ of fluid collection. KOA SF supernatant was collected by centrifugation at $16,000 \mathrm{x} \mathrm{g}$ at $4^{\circ} \mathrm{C}$ and stored at $-80^{\circ} \mathrm{C}$ until further analysis.

Cell culture and stimulation. 293T cells (American Type Culture Collection) were cultured in DMEM (Gibco; Thermo Fisher Scientific, Inc.) supplemented with $10 \%$ heat-inactivated fetal bovine serum (FBS; Gibco; Thermo Fisher Scientific, Inc.) and penicillin and streptomycin (Gibco; ThermoFisher Scientific,Inc.) at $37^{\circ} \mathrm{C}$ in a humidified incubator containing $5 \% \mathrm{CO}_{2}$. Peripheral blood mononuclear cells (PBMCs) were obtained from whole blood samples by density gradient centrifugation $(400 \mathrm{x} \mathrm{g}$ at room temperature for $30 \mathrm{~min}$ ) using Ficoll ${ }^{\circledR} 400$ (Sigma-Aldrich; Merck KGaA), according to the manufacturer's protocol. After washing twice with PBS, PBMCs were seeded $\left(3 \times 10^{6}\right.$ cells/well) into 12-well plates with RPMI-1640 (Gibco; Thermo Fisher Scientific, Inc.) containing $10 \% \mathrm{FBS}$ and $10 \mathrm{ng} / \mathrm{ml}$ macrophage colony-stimulating factor (R\&D Systems, Inc.). After incubation for 5 days at $37^{\circ} \mathrm{C}$, PBMC-derived macrophages were obtained, seeded $\left(1 \times 10^{6}\right.$ cells/well) into 12-well plates and co-incubated with $500 \mu$ l RPMI-1640-0.4\% BSA (Sigma-Aldrich; Merck KGaA) and $500 \mu \mathrm{l} \mathrm{SF}$ for 12,24 or $48 \mathrm{~h}$ at $37^{\circ} \mathrm{C}$. Control cells were treated with $1 \mathrm{ml}$ control medium (CM; RPMI-1640-0.4\% BSA). $\mathrm{CD}_{14}{ }^{+}$monocytes/macrophages $(\mathrm{MON} / \mathrm{Mc})$ were isolated from the KOA SF and PBMCs of patients with KOA or normal subjects using human CD14 MicroBeads (cat no. 130-050-201; MACS; Miltenyi Biotec, Inc.), according to the manufacturer's protocols. PBMC-derived macrophage apoptosis was induced by incubation with $1 \%$ DMSO (Sigma-Aldrich; Merck KGaA) for $12 \mathrm{~h}$ at $37^{\circ} \mathrm{C}$.

Cell transfection. PBMC-derived macrophages were seeded into 12 -well plates at a density of $1.5 \times 10^{5}$ cells per well and transfected with $100 \mathrm{nmol} / 1 \mathrm{miR}-155 \mathrm{mimic}$ (5'-UUAAUG CUAAUCGUGAUAGGGGU-3'), 100 nmol/l miR-155 inhibitor (5'-ACCCCUAUCACGAUUAGCAUUAA-3') or $100 \mathrm{nmol} / 1$ miR-155 mimic/inhibitor negative control (NC; 5'-UCACAA CCUCCUAGAAAGAGUAGA-3'; Shanghai GenePharma Co., Ltd.) using Lipofectamine ${ }^{\circledR} 2000$ reagent (Thermo Fisher Scientific, Inc.) according to the manufacturer's protocol. Following transfection for 24 or $48 \mathrm{~h}$ at $37^{\circ} \mathrm{C}$, PBMC-derived macrophages were co-incubated with $500 \mu \mathrm{l}$ RPMI-0.4\% BSA and $500 \mu \mathrm{l} \mathrm{SF}$ for a further $24 \mathrm{~h}$ at $37^{\circ} \mathrm{C}$. Control cells were treated with $1 \mathrm{ml} \mathrm{CM}$.

Reverse transcription-quantitative PCR (RT-qPCR). Total RNA was extracted from macrophages and 293 T cells used TRIzol ${ }^{\circledR}$ reagent (Thermo Fisher Scientific, Inc.). Total RNA was reverse transcribed into cDNA using the PrimeScript ${ }^{\mathrm{TM}}$ RT reagent kit (Takara Bio, Inc.), according to the manufacturer's protocol. Subsequently, cDNA was subjected to qPCR using SYBR ${ }^{\circledR}$ Premix Ex Taq ${ }^{\mathrm{TM}}$ (Takara Bio, Inc.). The thermocycling conditions were as follows: $5 \mathrm{~min}$ at $95^{\circ} \mathrm{C}$, followed by 40 cycles at $95^{\circ} \mathrm{C}$ for $15 \mathrm{sec}$ and $60^{\circ} \mathrm{C}$ for $30 \mathrm{sec}$. The primers used for qPCR are presented in Table I. mRNA and miRNA expression levels were quantified using the $2^{-\Delta \Delta \mathrm{Cq}}$ method (31) and normalized to the internal reference genes GAPDH and U6, respectively.

FCMstaining, analysis andapoptosisdetection.PBMC-derived macrophage single-cell suspensions $\left(1 \times 10^{6}\right.$ cells $\left./ 100 \mu \mathrm{l}\right)$ were incubated at $4^{\circ} \mathrm{C}$ for $30 \mathrm{~min}$ the with LIVE/DEAD ${ }^{\mathrm{TM}}$ Fixable Aqua Dead Cell Stain kit (Thermo Fisher Scientific, Inc.), which are a class of viability dyes suitable for identifying dead cells in samples that are sent for fixation. FcR-blocking reagent $\left(10 \mu \mathrm{l} ; 10 \mathrm{~min}\right.$ at $\left.4^{\circ} \mathrm{C}\right)$ and fluorescently labeled antibodies targeted against CD86 (1:100; cat. no. 12-0869-42; eBioscience; Fluorophores, R-phycoerythrin; Thermo Fisher Scientific, Inc.), inducible nitric oxide synthase (1:100; iNOS; cat. no. 53-5920-82; eBioscience; Fluorophores, Alexa Fluor 488; Thermo Fisher Scientific, Inc.) and CD206 (1:100; cat. no. 12-2069-41; eBioscience; Fluorophores: 
Table I. Primers used for quantitative PCR.

\begin{tabular}{|c|c|}
\hline Gene & Primer sequence $\left(5^{\prime} \rightarrow 3^{\prime}\right)$ \\
\hline IL-1 $\beta$ & $\begin{array}{ll}\text { F: } & \text { ATGATGGCTTATTACAGTGGCAA } \\
\text { R: } & \text { GTCGGAGATTCGTAGCTGGA }\end{array}$ \\
\hline IL-6 & $\begin{array}{ll}\mathrm{F}: & \text { ACTCACCTCTTCAGAACGAATTG } \\
\mathrm{R}: & \text { CCATCTTTGGAAGGTTCAGGTTG }\end{array}$ \\
\hline NOS2 & $\begin{array}{ll}\text { F: } & \text { TTCAGTATCACAACCTCA } \\
\text { R: } & \text { TGGACCTGCAAGTTAAAAT }\end{array}$ \\
\hline IL-10 & $\begin{array}{l}\text { F: GACTTTAAGGGTTACCTGGGTTG } \\
\text { R: }\end{array}$ \\
\hline ARG1 & $\begin{array}{l}\text { F: GTGGAAACTTGCATGGAC } \\
\text { R: AATCCTGGCACATCGGGAAT }\end{array}$ \\
\hline SOCS1 & $\begin{array}{l}\text { F: CACGCACTTCCGCACATTC } \\
\text { R: TAAGGGCGAAAAAGCAGTT }\end{array}$ \\
\hline CASP3 & $\begin{array}{ll}\text { F: } & \text { CATGGAAGCGAATCAATGGACT } \\
\text { R: } & \text { CTGTACCAGACCGAGATGTCA }\end{array}$ \\
\hline Ym1 & $\begin{array}{l}\text { F: } \text { TCACAAACAAAAGG } \\
\text { R: GAATATGTAACACATTCAA }\end{array}$ \\
\hline $\operatorname{miR}-155$ & $\begin{array}{ll}\text { F: } & \text { ATTGCCAATTTCTCTACCAC } \\
\text { R: } & \text { AGTAACAGGCATCATACACT }\end{array}$ \\
\hline GAPDH & $\begin{array}{l}\text { F: CAAGGTCATCCATGACAACTTTG } \\
\text { R: GTCCACCACCCTGTTGCTGTAG }\end{array}$ \\
\hline U6 & $\begin{array}{ll}\text { F: } & \text { CTCGCTTCGGCAGCACA } \\
\text { R: } & \text { AACGCTTCACGAATTTGCGT }\end{array}$ \\
\hline
\end{tabular}

IL, interleukin; NOS2, nitric oxide synthase 2; ARG1, arginase 1; SOCS1, suppressor of cytokine signaling 1; CASP3, caspase-3; $\mathrm{F}$, forward; $\mathrm{R}$, reverse.

PE-Cy7; Thermo Fisher Scientific, Inc.) were then added. The cell pellet was resuspended in $200 \mu \mathrm{l}$ PBS and reacted with Alexa Fluor ${ }^{\circledR}$ 488-labeled goat anti-mouse IgG antibody (1:200; Invitrogen; Thermo Fisher Scientific, Inc.) at room temperature for $30 \mathrm{~min}$ in darkness. Apoptotic cells were detected using the Annexin V/PI Cell Apoptosis kit (Sungene Biotech Co., Ltd.), according to the manufacturer's protocol. FCM analyses were performed using a flow cytometer (BD Accuri C6 cytometer; BD Biosciences) and FlowJo software (version 7.6.1; FlowJo LLC).

Western blotting. Total protein was isolated from macrophages and cells using protein extraction kit (Bio-Rad Laboratories, Inc.), after which protein concentration was determined by a BCA assay (Beyotime Institute of Biotechnology). Subsequently, samples were separated by $8-12 \%$ SDS-PAGE and transferred onto nitrocellulose membranes using a semidry transfer apparatus. PVDF membranes were washed with TBST (0.05\% Tween-20) three times before being blocked with $5 \%$ skimmed milk at room temperature for $2 \mathrm{~h}$. The membranes were then incubated at $4^{\circ} \mathrm{C}$ overnight with primary antibodies targeted against: Phosphorylated (p)-STAT1 (1:1,000; cat. no. ab30645; Abcam), STAT1 (1:1,000; cat. no. ab2415; Abcam), p-STAT6 (1:1,000; cat. no. ab54461; Abcam), STAT6 (1:1,000; cat. no. ab44718; Abcam), SOCS1 (1:1,000; cat. no. 3950; Cell Signaling Technology, Inc.), caspase-3 (CASP3) (1:1,000; cat. no. 9662; Cell Signaling Technology, Inc.) and GAPDH (1:1,000; cat. no. 60004-1-Ig; ProteinTech, Inc.). The membranes were washed with TBST three times, incubated with horseradish peroxidase-conjugated goat anti-rabbit (1:1,000; cat. no. ab6721; Abcam) and goat anti-mouse (cat. no. ab97040; 1:5,000; Abcam) secondary antibodies for $2 \mathrm{~h}$ at room temperature. ECL chemiluminescence solution and bicinchoninic acid protein quantitative detection kits were purchased from Beyotime Institute of Biotechnology. Finally, ImageJ (version 1.38; National Institutes of Health) was used to analyze the gray value.

Luciferasereporterassay. TheinteractionsitesbetweenmiR-155-5p and CASP3 were predicted using miRanda (http://www. microrna.org), miRwalk (http://mirwalk.umm.uniheidelberg.de) and miRTarbase (http://mirtarbase.mbc.nctu.edu.tw) databases. The online resource microRNA.org (http://www.microrna.org) predicted the targeted binding site between miR-155-5p and the 3'-untranslated region (3'-UTR) SOCS1. The wild-type (WT) or mutant (MUT) 3'-UTR of SOCS1 or CASP3 (constructed by Guangzhou RiboBio Co., Ltd.) were cloned into the pMIR vector (Guangzhou RiboBio Co., Ltd.). 293T cells were seeded $\left(4 \times 10^{5}\right.$ cells/well) into plates with $0.5 \mathrm{ml}$ complete growth medium. At 70-90\% confluency, 293T cells were co-transfected with the miR-155 mimic or miR-155 inhibitor, negative control (miR-NC) and $50 \mathrm{ng}$ pMIR-SOCS1-3'-UTR-WT or pMIR-SOCS1-3'-UTR-MUT using Lipofectamine $3000^{\circledR}$ reagent (Thermo Fisher Scientific, Inc.). For each well, $1 \mu \mathrm{g} / \mu 1$ DNA was diluted in $50 \mu$ l Opti-MEM (Gibco; Thermo Fisher Scientific, Inc.), whilst in a separate tube, $2.5 \mu 1$ Lipofectamine ${ }^{\circledR} 3000$ was diluted in $50 \mu \mathrm{l}$ Opti-MEM. Each tube was gently mixed and incubated for $20 \mathrm{~min}$ at room temperature. Subsequently, the two solutions were added to each well and gently mixed. Cells were incubated for $48 \mathrm{~h}$ before assessing transfection efficiency. The same operating procedures were used for the miR-155-5p/CASP3 luciferase reporter assay using pMIR-CASP3-3'-UTR-WT and pMIR-CASP3-3'-UTR-MUT vectors. At $48 \mathrm{~h}$ post-transfection, cells were harvested and luciferase activities were measured using the Dual-Luciferase ${ }^{\circledR}$ Reporter System (Promega Corporation), according to the manufacturer's protocol. Firefly luciferase activity was normalized to that of Renilla luciferase.

Statistical analysis. Statistical analyses were performed using GraphPad Prism software (version 7; GraphPad Software Inc.). Data are presented as the mean \pm standard deviation. $\mathrm{P}<0.05$ was considered to indicate a statistically significant difference. Comparisons among groups were analyzed using one-way ANOVA followed by Tukey's post hoc test.

\section{Results}

KOA SF promotes PBMC-derived M1 macrophage polarization and inhibits macrophage apoptosis. The effects of SF from patients with KOA on PBMC-derived macrophages were assessed using RT-qPCR and FCM. KOA SF significantly promoted the expression of M1-type markers by the macrophages (Fig. 1A), whilst partially reducing the expression of M2-type markers (Fig. 1B) compared with macrophages incubated in CM. The FCM results indicated that SF significantly 
A

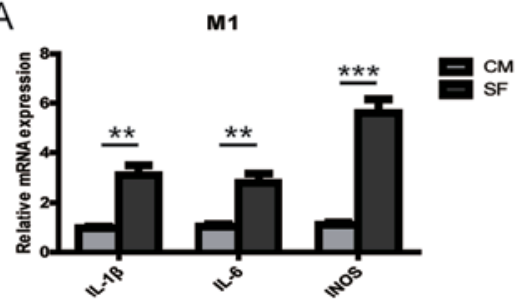

C
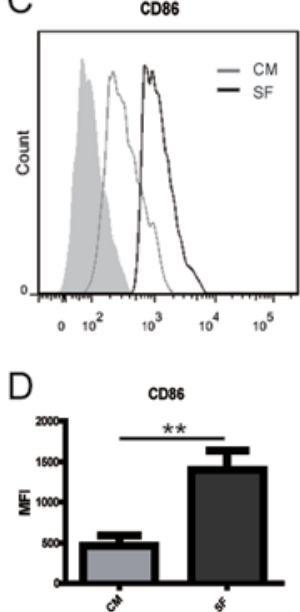

$\mathrm{B}$

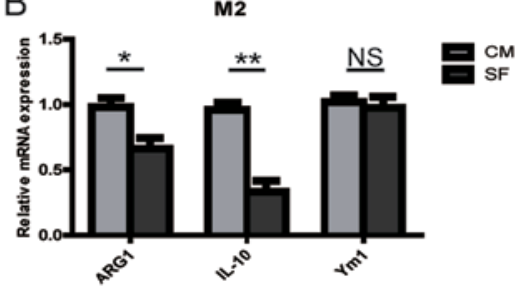

E
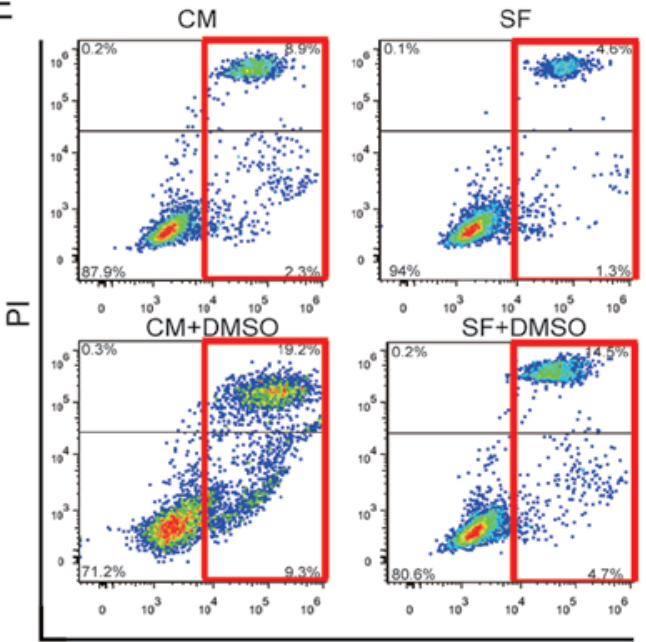

INOS
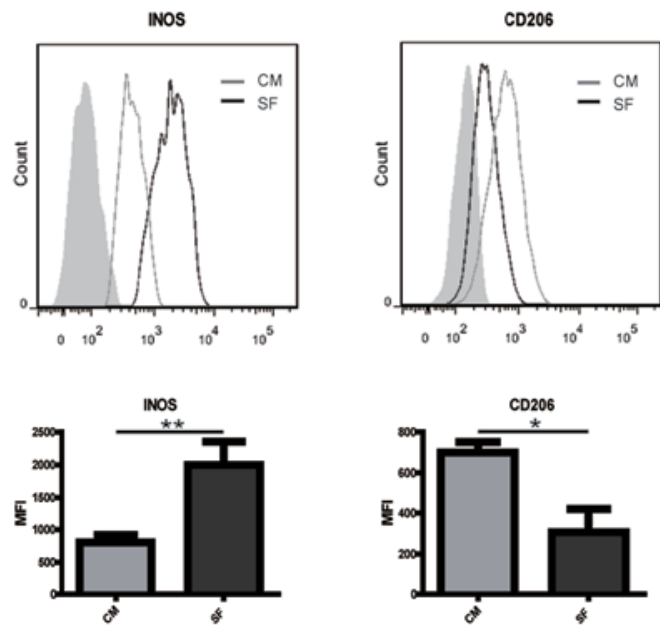

$\mathrm{F}$

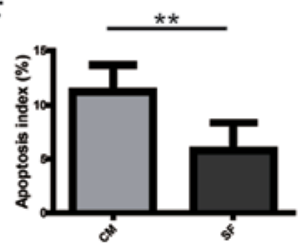

Annexin- $V$
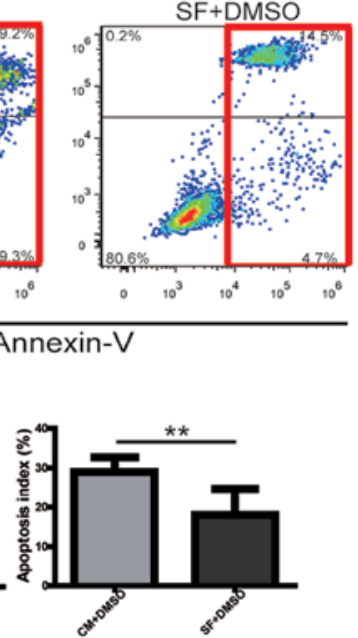

Figure 1. KOA SF promotes PBMC-derived macrophage M1 polarization and inhibits macrophage apoptosis. The relative expression of (A) M1 and (B) M2 markers in PBMC-derived macrophages cultured with either KOA SF or CM for $12 \mathrm{~h}$. CD86, iNOS and CD206 expression levels in PBMC-derived macrophages cultured with KOA SF or CM for $24 \mathrm{~h}$ were (C) determined by flow cytometry and (D) quantified. The grey shadow represents the isotype control. Following treatment with or without $1 \%$ DMSO for $12 \mathrm{~h}$ in RPMI-1640 containing $10 \%$ FBS, PBMC-derived macrophages were cultured with KOA SF or $\mathrm{CM}$ for $24 \mathrm{~h}$. Apoptotic cells were $(\mathrm{E})$ detected by flow cytometry (red box indicates apoptotic cells) and $(\mathrm{F}) \mathrm{quantified}$. ${ }^{*} \mathrm{P}<0.05$, ${ }^{* *} \mathrm{P}<0.01$ and ${ }^{* * * *} \mathrm{P}<0.001$ KOA, knee osteoarthritis; SF, synovial fluid; PBMC, peripheral blood mononuclear cells; CM, control medium; iNOS, inducible nitric oxide synthase; IL, interleukin; ARG1, arginase 1; Ym1, chitinase-like 3; NS, not significant; PI, propidium iodide.

promoted the expression of CD86 and iNOS, whilst reducing the expression of CD206 in PBMC-derived macrophages compared with those incubated with CM (Fig. 1C and D). During OA, cartilage loses its integrity, where the number of apoptotic chondrocytes and immune cells increases (6). The mechanism underlying this increase in the presence of KOA SF was therefore investigated by assessing its effects on macrophage apoptosis. In the presence and absence of $1 \%$ DMSO, KOF SF inhibited PBMC-derived macrophage apoptosis compared with those incubated with CM (Fig. 1E and F). The results suggested that the increase in M1 macrophages in KOA SF may be caused by the proinflammatory SF during the progression of OA.

Expression of miR-155-5p is upregulated in KOA SF-derived macrophages and KOA SF-cultured PBMC-derived macrophages. A recent study reported that miR-155-5p was associated with OA (21). The expression level of miR-155-5p was found to be upregulated in $\mathrm{CD} 14^{+} \mathrm{MON} / \mathrm{Mc}$ derived from the SF of patients with KOA compared with that in $\mathrm{CD} 14^{+}$ $\mathrm{MON} / \mathrm{Mc}$ isolated from the PBMCs of patients with KOA or healthy individuals (Fig. 2A). In addition, the expression of SOCS1, a target of miR-155-5p, was revealed to be downregulated in $\mathrm{CD}_{1} 4^{+} \mathrm{MON} / \mathrm{Mc}$ derived from the $\mathrm{SF}$ of patients with KOA compared with $\mathrm{CD} 14^{+} \mathrm{MON} / \mathrm{Mc}$ isolated from the PBMCs of patients with KOA or healthy individuals (Fig. 2B). PBMC-derived macrophages cultured in KOA SF expressed significantly higher levels of miR-155-5p compared with macrophages cultured in CM (Fig. 2C). These results suggested that miR-155-5p may serve an important role in polarizing macrophages during OA progression.

KOA SF-induced promotion of PBMC-derived M1 macrophage polarization is associated with the miR-155-5p/SOCS1 signaling pathway. As a potential target of miR-155-5p, SOCS1-mediated inhibition of p-STAT1 activity and promotion of p-STAT6 activity to induce macrophage M2 polarization whilst inhibiting M1 macrophage polarization has been extensively studied in RAW264.7 cells (16). Therefore, it was hypothesized that miR-155-p serves a role in KOA-SF-induced PBMC-derived M1 macrophage polarization via SOCS1. Transfection with the miR-155-5p mimic significantly increased miR-155-5p expression, whilst transfection with the miR-155-5p inhibitor significantly reduced miR-155-5p expression in PBMC-derived macrophages (Fig. 2D). Following transfection with NC or miR-155-5p mimic for $24 \mathrm{~h}, \mathrm{PBMC}$-derived macrophages were cultured with $\mathrm{CM}$ or KOA SF. The expression of miR-155-5p in PBMC-derived macrophages transfected with miR-155-5p mimic was upregulated compared with the CM NC group, while the upregulation of miR-155-5p expression in PBMC-derived macrophages cultured with KOA SF was not significantly different compared with PBMC-derived macrophages cultured with CM (Fig. 2E). Additionally, RT-qPCR results indicated that SOCS1 expression was significantly increased in the SOCS1-WT and SOCS1-MUT groups compared with that in 

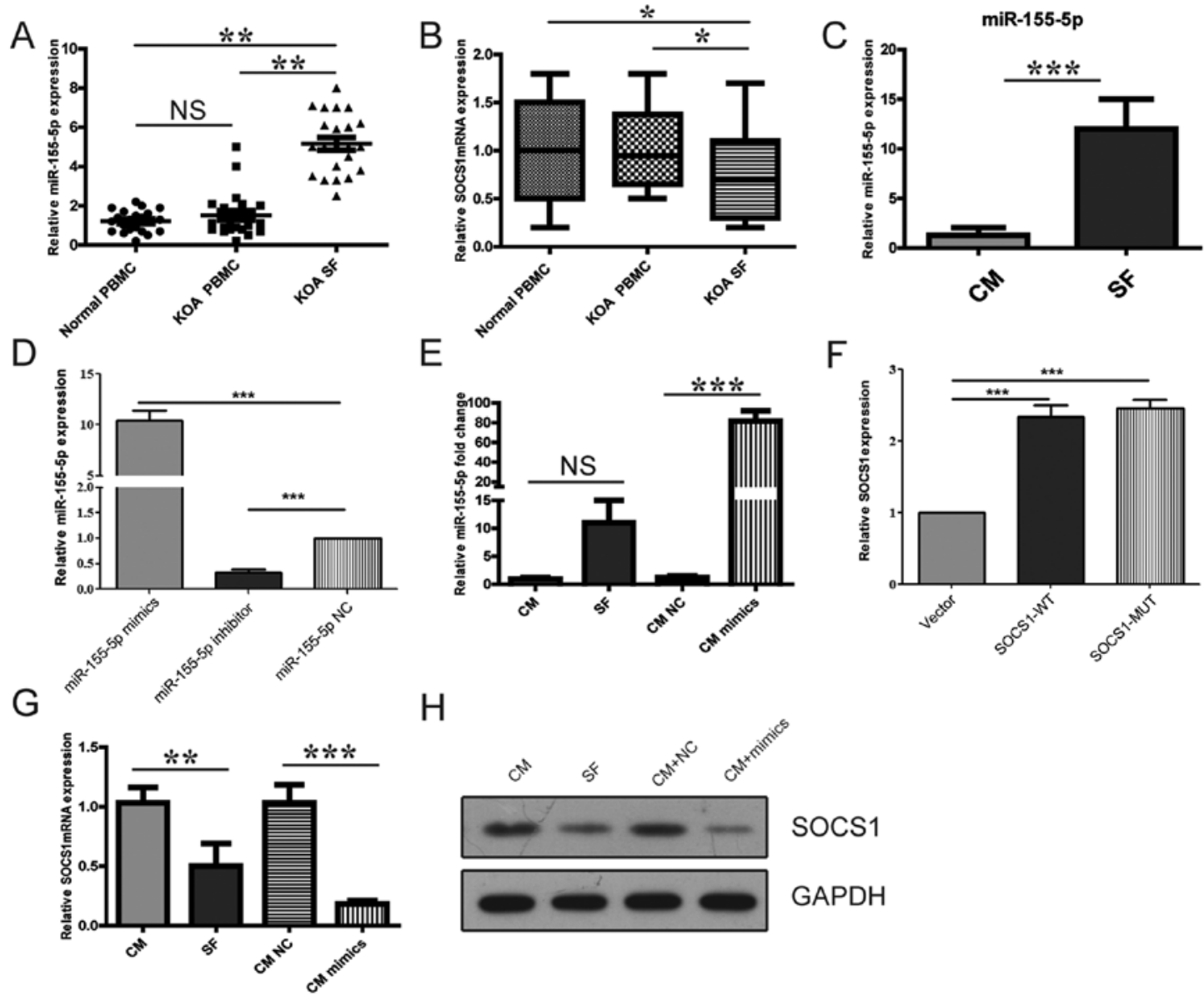

$\mathrm{E}$

$\mathrm{F}$
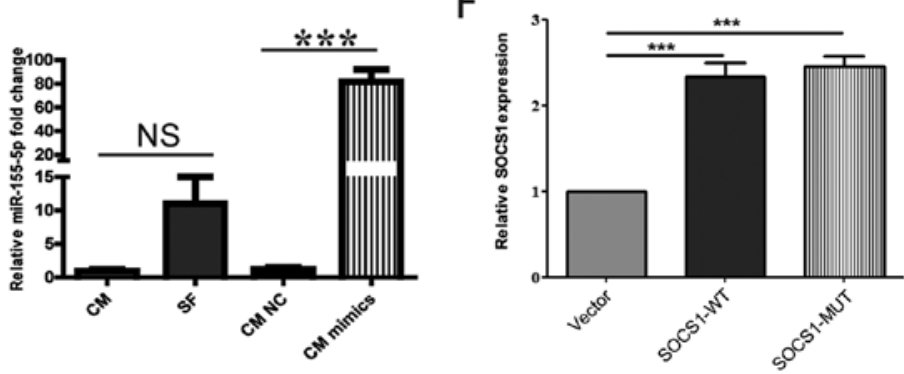

$\mathrm{H}$

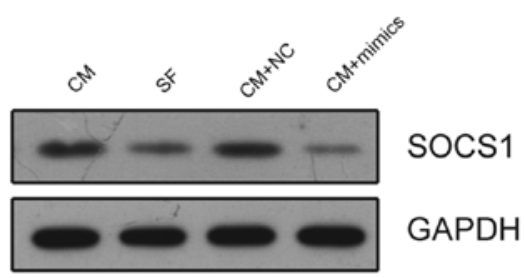

I

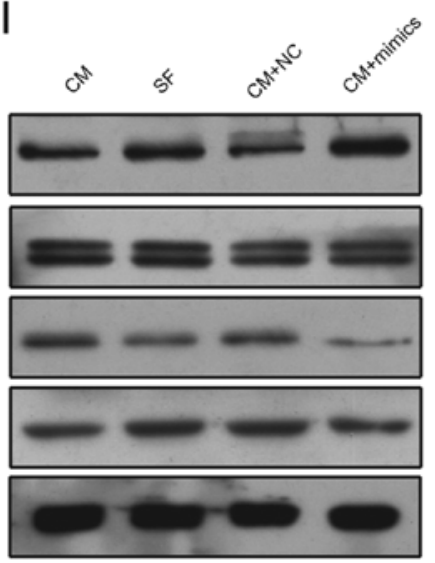

p-STAT1

J 5' CgCCGUG-CACG--CAGCAUUAa $3^{\prime}$ SOCS1 3'UTR WT

3' uggGgauagugcuaAucguaAu 5 ' miR-155-5p

5. II I: II IICCGUG-UGCG--CCUACUUAa $3^{\prime}$ SOCS1 3 ' UTR MUT

STAT1
p-STAT6
STAT6
GAPDH

K

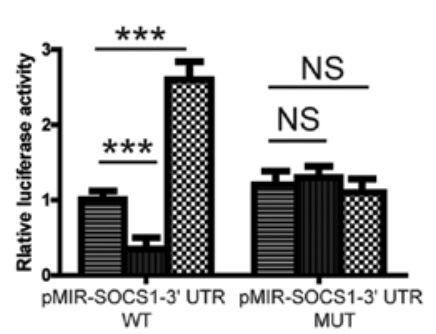

NC

miR-155-5p mimics miR-155-5p inhibitor

Figure 2. M1 polarization is associated with the miR-155-5p/SOCS1 signaling pathway. The relative expression of (A) miR-155-5p and (B) SOCS1 in PBMC-derived macrophages isolated from the blood samples of normal individuals, patients with KOA and PBMC-derived macrophages isolated from the KOA SF. (C) The expression of miR-155-5p in PBMC-derived macrophages cultured with KOA SF or CM for $12 \mathrm{~h}$ was detected by reverse transcription-quantitative PCR. The relative expression of miR-155-5p (D) in cells transfected with either NC, miR-155-5p mimic or the miR-155-5p inhibitor, (E) in transfected cells cultured with CM or KOA and in cells transfected with either NC or miR-155-5p cultured in CM. (F) The relative expression of SOCS1 in PBMC-derived macrophages transfected with control vector, SOCS-WT or SOCS-MUT. The relative expression of SOCS1 (G) mRNA and (H) protein in PBMC-derived macrophages treated and/or transfected with CM, KOA SF, CM + NC or CM + miR-155-5p mimic. (I) Levels of STAT1 and STAT6 phosphorylation in PBMC-derived macrophages treated and/or transfected with CM, KOA SF, CM + NC or CM + miR-155-5p mimic. (J) The potential interaction between miR-155-5p and SOCS1 3'-UTR-WT or SOCS1 3'-UTR-MUT. (K) The interaction between miR-155 and SOCS1 was confirmed using a dual-luciferase reporter assay. "P<0.05, ${ }^{* *} \mathrm{P}<0.01$ and ${ }^{* * *} \mathrm{P}<0.001$. miR, microRNA; SOCS1, suppressor of cytokine signaling 1; KOA, knee osteoarthritis; SF, synovial fluid; PBMC, peripheral blood mononuclear cells; CM, control medium; NC, negative control; p, phosphorylated; 3'-UTR, 3'-untranslated region; WT, wild-type; MUT, mutant; NS, not significant.

the control vector group (Fig. 2F). The expression of SOCS1 mRNA and protein in PBMC-derived macrophages cultured with KOA SF or transfected with the miR-155-5p mimic was found to be downregulated compared with that in the corresponding control groups (Fig. $2 \mathrm{G}$ and $\mathrm{H}$ ). In addition, the activation of STAT1 was revealed to be increased whereas the activation of STAT6 was found to be reduced in PBMC-derived macrophages cultured with KOA SF or transfected with miR-155-5p mimic compared with those in the corresponding control groups (Fig. 2I). The online resource microRNA.org 

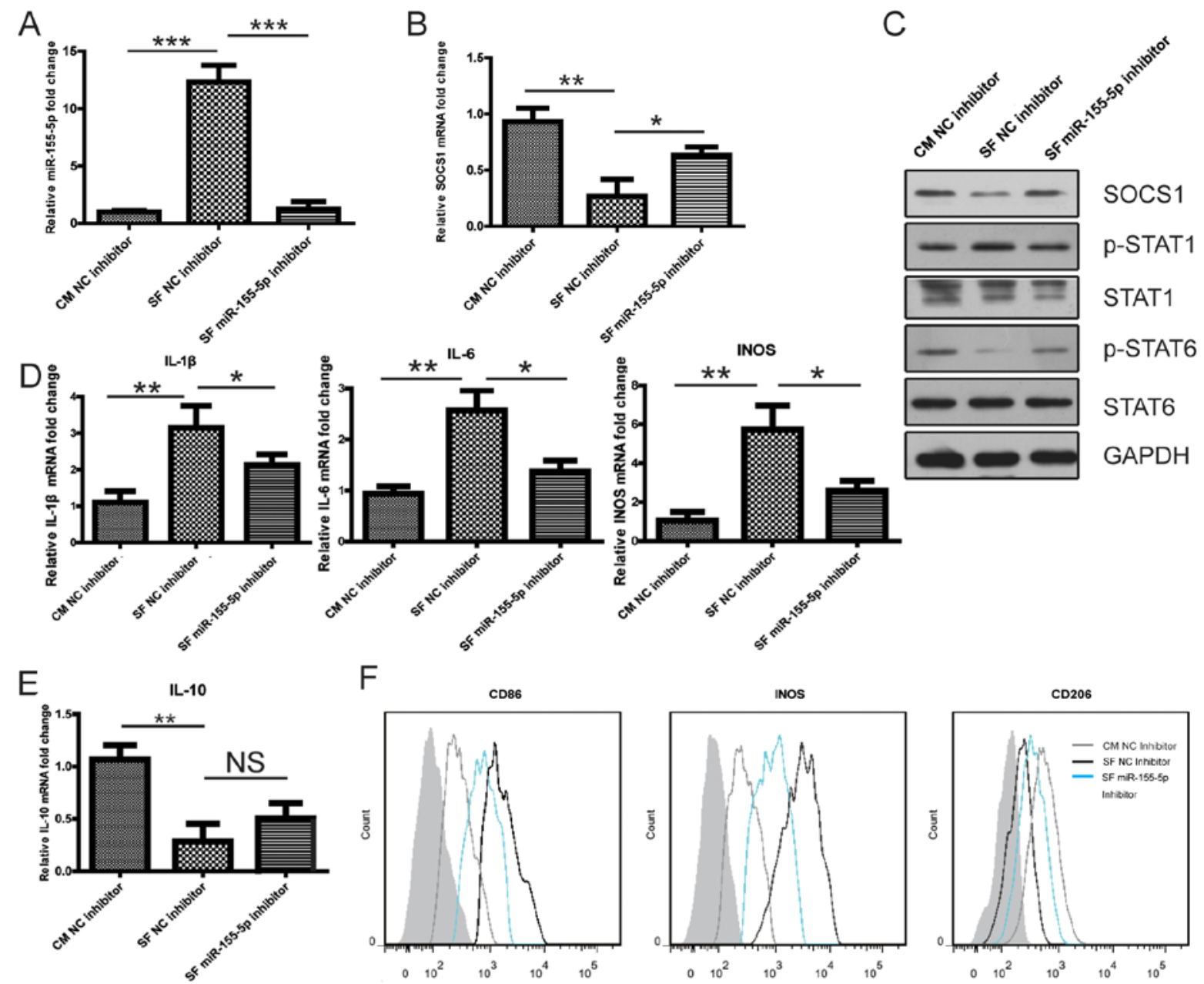

$\mathrm{F}$

G
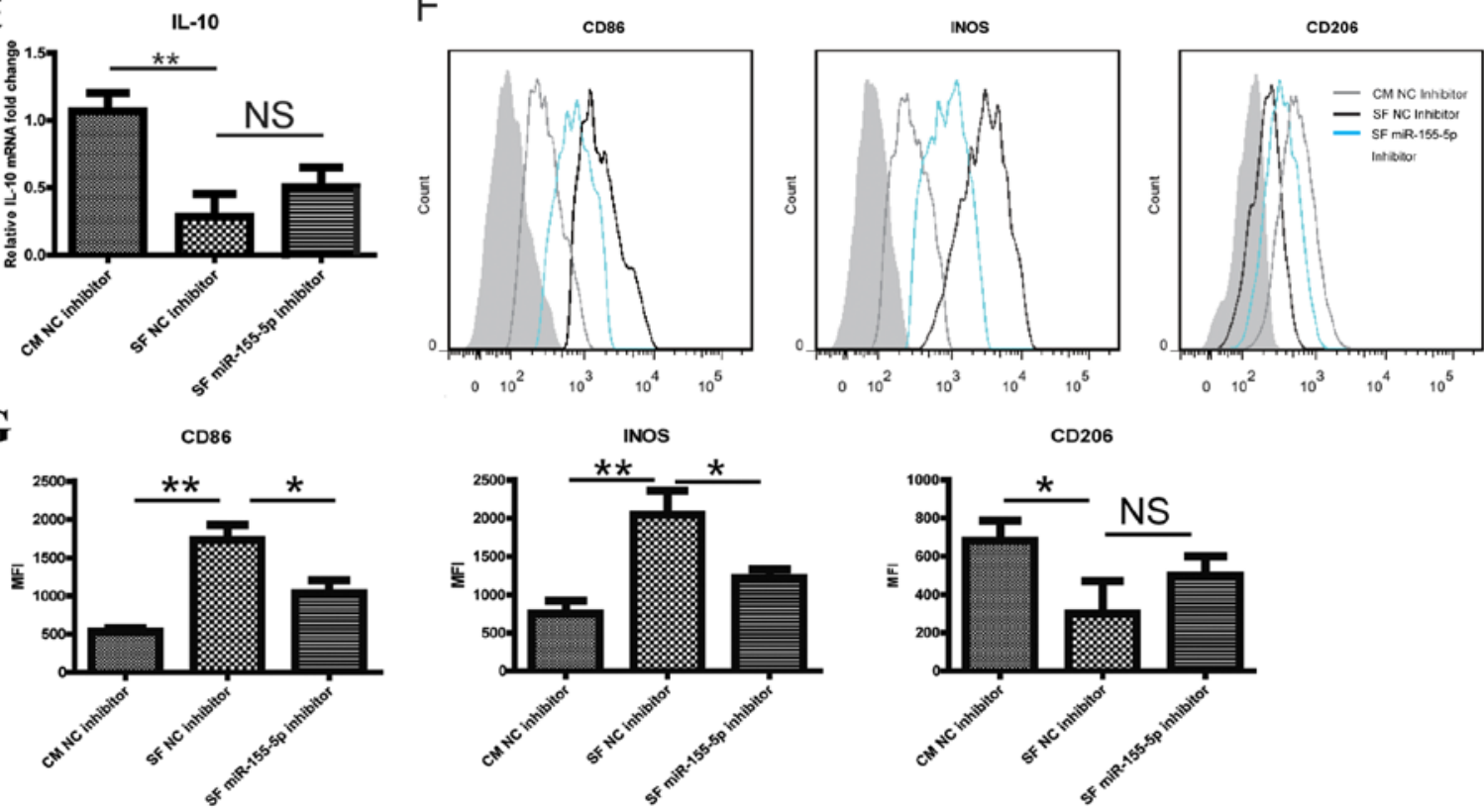

Figure 3. miR-155-5p downregulation promotes SOCS1 expression. (A-G) Following transfection with the NC inhibitor or miR-155-5p inhibitor for $24 \mathrm{~h}$, PBMC-derived macrophages were cultured with CM or KOA SF. The relative expression of (A) miR-155-5p and (B) SOCS1 mRNA in PBMC-derived macrophages. (C) SOCS1 protein expression and the phosphorylation levels of STAT1 and STAT6 in PBMC-derived macrophages. The relative expression of (D) IL-1 $\beta$, IL-6, iNOS and (E) IL-10 mRNA in PBMC-derived macrophages. The expression levels of CD86, iNOS and CD206 expression in PBMC-derived macrophages was (F) determined by flow cytometry and (G) quantified. The grey shadow represents the isotype control. ${ }^{*} \mathrm{P}<0.05,{ }^{* *} \mathrm{P}<0.01$ and ${ }^{* * *} \mathrm{P}<0.001$, as indicated. miR, microRNA; SOCS1, suppressor of cytokine signaling 1; NC, negative control; PBMC, peripheral blood mononuclear cells; CM, control medium; KOA, knee osteoarthritis; SF, synovial fluid; p, phosphorylated; IL, interleukin; iNOS, inducible nitric oxide synthase; NS, not significant.

(http://www.microrna.org) predicted the targeted binding site between miR-155-5p and the 3'-UTR SOCS1. Subsequently, dual-luciferase reporters encoding the WT or MUT 3'-UTR of SOCS1 were constructed (Fig. 2J). Co-transfection with the miR-155-3p mimic significantly inhibited the dual-luciferase activity of the SOCS1 WT 3'-UTR SOCS1 plasmid but not that of the SOCS1 MUT 3'-UTR plasmid. By contrast, the miR-155-5p inhibitor significantly enhanced the dual-luciferase activity of the SOCS1 WT 3'-UTR plasmid but not that of the SOCS1 MUT 3'-UTR (Fig. 2K). These results suggested a regulatory relationship between miR-155 and SOCS1 mRNA.
Downregulation of miR-155-5p promotes SOCS1 expression and inhibits M1 polarization of KOA SF-stimulated $P B M C$-derived macrophages. Following transfection with the $\mathrm{NC}$ inhibitor or miR-155-5p inhibitor for $24 \mathrm{~h}$, PBMC-derived macrophages were cultured with $\mathrm{CM}$ or KOA SF. The downregulation of miR-155-5p reversed the reduced SOCS1 mRNA and protein expression by KOA SF (Fig. 3A-C). Furthermore, PBMC-derived macrophages cultured in KOA SF exhibited increased p-STAT1 and decreased p-STAT6 levels compared with those in the CM group. However, in PBMC-derived macrophages transfected with the miR-155-5p inhibitor and cultured 


\section{A}

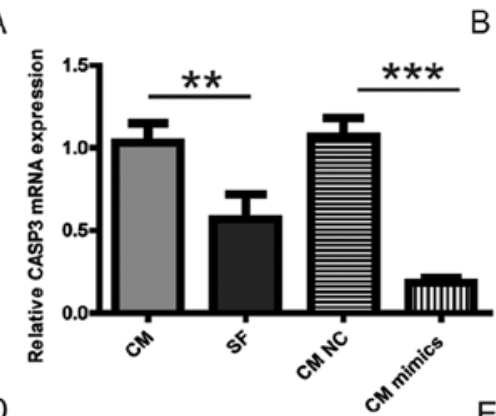

$\mathrm{B}$

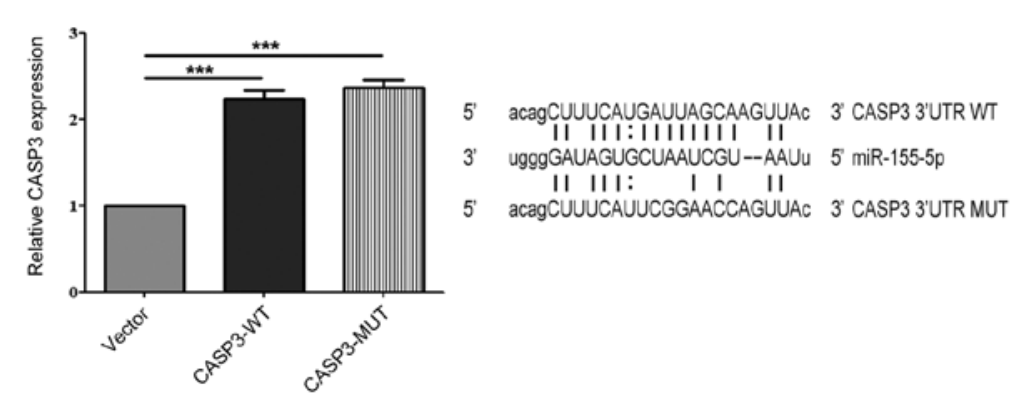

C

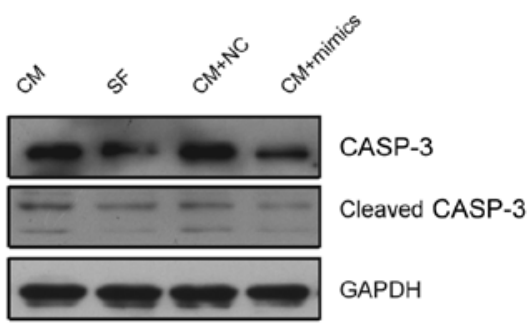

F

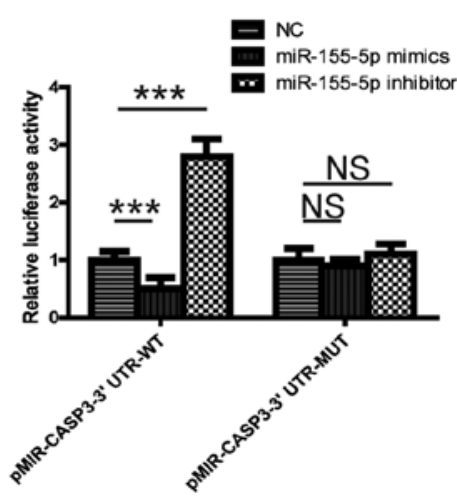

Figure 4. PBMC-derived macrophages are regulated by the miR-155-5p/CASP3 signaling pathway. (A-C) Following transfection with NC or miR-155-5p mimic for $24 \mathrm{~h}$, PBMC-derived macrophages were cultured with CM or KOA SF. The relative expression of CASP3 (A) mRNA and (B) protein. (C) The relative expression of CASP3 and cleaved CASP3 in PBMC-derived macrophages treated with 1\% DMSO. (D) The relative expression of CASP3 following transfection with the control vector, CASP-WT or CASP-MUT. (E) The binding sites of miR-155-p on CASP3 3'-UTR-WT or CASP3 3'-UTR-MUT. (F) The potential interaction between miR-155 and CASP3 was verified using a dual-luciferase reporter assay., ${ }^{* *} \mathrm{P}<0.01$ and ${ }^{* * *} \mathrm{P}<0.001$. PBMC, peripheral blood mononuclear cells; miR, microRNA; CASP3, caspase-3; NC, negative control; CM, control medium; KOA, knee osteoarthritis; SF, synovial fluid; 3'-UTR, 3'-untranslated region; WT, wild-type; MUT, mutant; NS, not significant.

in KOA SF, the KOA SF-induced effects on STAT1 and STAT6 phosphorylation were reversed (Fig. 3C). In PBMC-derived macrophages transfected with the miR-155-5p inhibitor, the KOA SF-induced increased expression of IL-1 $\beta$, IL-6, iNOS and CD86 was reversed, however the reduced expression of IL-10 and CD206 as a result of KOA-SF treatment was not reversed (Fig. 3D, E, F and G). These observations indicated further that KOA SF promoted M1 macrophage polarization by increasing miR-155-5p, which targeted SOCS1 to upregulate p-STAT1 activity whilst inhibiting p-STAT6 activity.

KOA SF-induced inhibition of PBMC-derived macrophage apoptosis occurs viathe miR-155-5p/CASP3 signaling pathway. miR-155-5p has been previously associated with apoptosis in several cancer cell lines and carcinomas (23); therefore, it was hypothesized that miR-155-5p may serve an important role in KOA SF-induced suppression of PBMC-derived macrophage apoptosis. The online microRNA, miRanda (http://www. microrna.org), miRwalk (http://mirwalk.umm.uniheidelberg. de) and miRTarbase (http://mirtarbase.mbc.nctu.edu.tw) databases predicted that CASP3 was a target gene of miR-155-5p, whilst the microRNA.org database identified the target binding site for miR-155 on the 3'-UTR of CASP3 mRNA (Fig. 4E). The expression of CASP3 mRNA was found to be significantly reduced in PBMC-derived macrophages cultured with KOA SF or transfected with miR-155-5p mimic compared with that in the corresponding control groups (Fig. 4A). The expression of CASP3 and cleaved CASP3 were also revealed to be decreased in PBMC-derived macrophages cultured with KOA SF or transfected with miR-155-5p mimic compared with those in the corresponding control groups (Fig. 4B and C). Additionally, the RT-qPCR results indicated that CASP3 expression was significantly increased in CASP3-WT and CASP3-MUT groups compared with that in the control vector group (Fig. 4D). To further investigate the interaction between miR-155-5p and CASP3, WT and MUT CASP3 3'-UTRs were cloned into the pMIR-reporter plasmid (Fig. 4E). The dual-luciferase reporter assay indicated that the luciferase activity of pMIR-CASP3-3'-UTR-WT, but not pMIR-CASP3-3'-UTR-MUT, was significantly decreased by co-transfection with the miR-155-5p mimic and significantly enhanced by transfection with the miR-155-5p inhibitor compared that in the miR-NC group (Fig. 4F). These findings indicated that KOA SF increased miR-155-5p-induced inhibition of macrophage apoptosis by targeting CASP3.

miR-155-5p downregulation promotes CASP3 expression and enhances macrophage apoptosis. Following transfection with the NC inhibitor or miR-155-5p inhibitor for $24 \mathrm{~h}$, PBMC-derived macrophages were cultured with $\mathrm{CM}$ or KOA SF. miR-155-5p downregulation was found to reverse the KOA SF-induced downregulation of CASP3 mRNA and protein expression (Fig. 5A and B). Furthermore, in 1\% DMSO-treated PBMC-derived macrophages, KOA SF reduced the expression of CASP3 and cleaved CASP3 (Fig. 5C). However, in PBMC-derived macrophages transfected with the miR-155-5p inhibitor, the reduced expression of CASP3 and cleaved CASP3 by KOA SF was abolished (Fig. 5C). In addition, both in the presence and absence of $1 \%$ DMSO treatment, KOA SF treatment suppressed PBMC-derived macrophage apoptosis, 

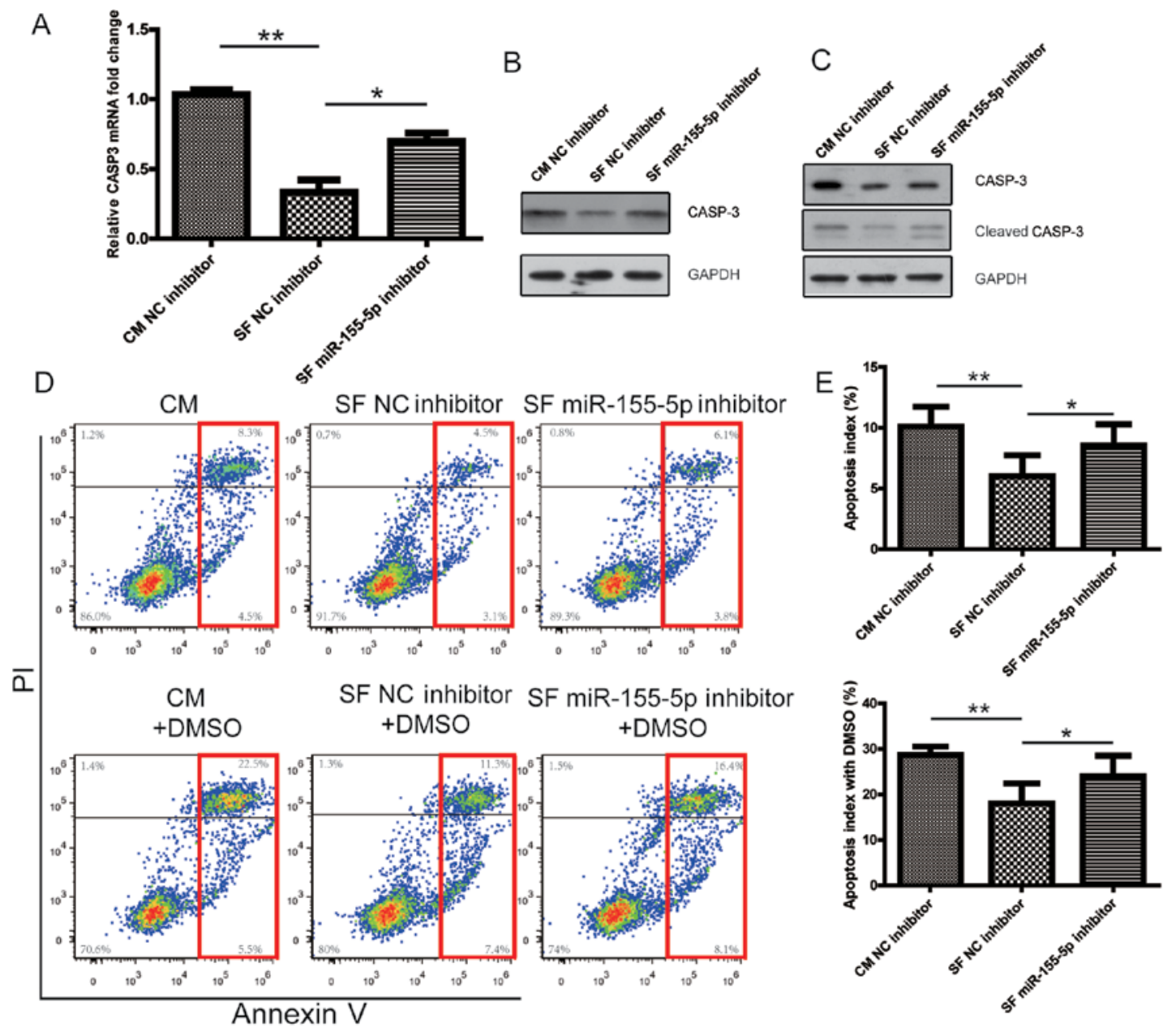

Figure 5. Downregulation of miR-155-5p promotes CASP3 expression and enhances macrophage apoptosis. (A-E) Following transfection with NC or miR-155-5p inhibitor for $24 \mathrm{~h}$, PBMC-derived macrophages were cultured with CM or KOA SF. The relative expression of CASP3 (A) mRNA and (B) in PBMC-derived macrophages. (C) The relative expression of CASP3 and cleaved CASP3 in PBMC-derived macrophages treated with 1\% DMSO. PBMC-derived macrophage apoptosis following treatment with or without $1 \%$ DMSO was (D) determined by flow cytometry (red box indicates apoptotic cells) and (E) quantified. ${ }^{*} \mathrm{P}<0.05$ and ${ }^{* * *} \mathrm{P}<0.01$. miR, microRNA; CASP3, caspase-3; NC, negative control; CM, control medium; KOA, knee osteoarthritis; SF, synovial fluid; PBMC, peripheral blood mononuclear cells; PI, propidium iodide.

which was reversed by transfection with the miR-155-5p inhibitor (Fig. 5D and E). These results indicated that CASP3 is a novel target of miR-155-5p and that KOA SF inhibited macrophage apoptosis via the miR-155-5p/CASP3 signaling pathway.

\section{Discussion}

OA is a debilitating condition that affects millions of individuals worldwide, where the only effective treatment strategies available are knee and hip replacement surgeries (2). Therefore, identifying the mechanism underlying $\mathrm{OA}$ is of substantial importance. OA has a complex pathogenesis where its etiology is remains to be completely elucidated (1). A previous study has revealed that macrophages may serve as a major factor in the acceleration of OA progression (7). In particular, miR-155-5p has been previously found to associate with $\mathrm{OA}$, where increased miRNA-155-5p expression leads to the M1 polarization of the RAW 264.7 macrophage cell line (27). It has also been reported that miR-155 is involved in TNF- $\alpha$-mediated inhibition of osteogenesis differentiation, which directly targets a suppressor of cytokine signaling 1 (SOCS1) to inhibit bone morphogenetic protein 2-induced osteoblast differentiation (32). In the present study, PBMC-derived macrophages cultured in KOA SF displayed upregulated expression of M1 markers, reduced apoptosis and increased miR-155-5p expression compared with those cultured in CM. The present study investigated the relationship between macrophage phenotype, apoptosis and KOA. The results indicated that the proinflammatory microenvironment of KOA SF may favor M1 macrophage polarization by upregulating miR-155-5p whilst inhibiting M1 macrophage apoptosis. Therefore, the results of the present study may provide an explanation for the increased ratio of total to M1-like macrophages in the granulocyte cell population during KOA progression. In addition, the results of the present study suggested that KOA SF altered PBMC-derived macrophages M1 polarization via the miR-155-5p/SOCS1 signaling pathway. CASP3 was subsequently identified as a novel target of miR-155-5p, where the results indicated that KOA SF inhibited macrophage apoptosis through the miR-155-5p/CASP3 signaling pathway. Based on the results of the aforementioned study (33), the present study suggested that KOA SF promoted PBMC-derived M1 macrophage polarization via the miR-155-5p/SOCS1 signaling pathway and inhibited macrophage apoptosis via the same signaling pathway. 
It should be noted that the present study has a number of limitations. Since the infiltration of MON/Mcs in patients with OA was not investigated, the possibility that MON/Mc infiltration may affect the ratio of macrophages in the granulocyte cell population cannot not be ruled out. In addition, miR-155-5p was one of the most important elements that affected macrophage polarization phenotype and apoptosis in KOA SF vs. CM, but other factors can be involved. Further studies are required to explore the mechanism underlying the effect of the KOA SF microenvironment on macrophage polarization, phenotype and apoptosis. Based on the results of the present study, it was hypothesized that targeting the inflammatory KOA SF and macrophage polarization may delay or prevent OA progression. Further investigation into the potential of miR-155-5p as a therapeutic target for KOA is required.

\section{Acknowledgements}

Not applicable.

\section{Funding}

The present study was supported by the Natural Science Joint Special Foundation of Shandong Province (grant no. 20160841).

\section{Availability of data and materials}

The datasets used and/or analyzed during the current study are available from the corresponding author on reasonable request.

\section{Authors' contributions}

GDW designed the study, collected the data and wrote the manuscript. GSL and LC performed the experiments and interpreted the data. All authors read and approved the final manuscript.

\section{Ethics approval and consent to participate}

The present study was approved by the Ethics Committee of the Yantai Yuhuangding Hospital (Yantai, China). Written informed consent was obtained from all patients.

\section{Patient consent for publication}

Not applicable.

\section{Competing interests}

The authors declare that they have no competing interests.

\section{References}

1. Glyn-Jones S, Palmer AJ, Agricola R, Price AJ, Vincent TL, Weinans H and Carr AJ: Osteoarthritis. Lancet 386: 376-387, 2015.

2. Losina E, Weinstein AM, Reichmann WM, Burbine SA, Solomon DH, Daigle ME, Rome BN, Chen SP, Hunter DJ, Suter LG, et al: Lifetime risk and age at diagnosis of symptomatic knee osteoarthritis in the US. Arthritis Care Res (Hoboken) 65 : 703-711, 2013.
3. Boilard E, Nigrovic PA, Larabee K, Watts GF, Coblyn JS, Weinblatt ME, Massarotti EM, Remold-O'Donnell E, Farndale RW, Ware J, et al: Platelets amplify inflammation in arthritis via collagen-dependent microparticle production. Science 327: 580-583, 2010.

4. Kriegova E, Manukyan G, Mikulkova Z, Gabcova G, Kudelka M, Gajdos P and Gallo J: Gender-related differences observed among immune cells in synovial fluid in knee osteoarthritis. Osteoarthritis Cartilage 26: 1247-1256, 2018.

5. Pers YM, Quentin J, Feirreira R, Espinoza F, Abdellaoui N, Erkilic N, Cren M, Dufourcq-Lopez E, Pullig O, Nöth U, et al: Injection of adipose-derived stromal cells in the knee of patients with severe osteoarthritis has a systemic effect and promotes an anti-inflammatory phenotype of circulating immune cells Theranostics 8: 5519-5528, 2018.

6. Hunter DJ and Bierma-Zeinstra S: Osteoarthritis. Lancet 393: 1745-1759, 2019.

7. Kraus VB, McDaniel G, Huebner JL, Stabler TV, Pieper CF Shipes SW, Petry NA, Low PS, Shen J, McNearney TA, et al: Direct in vivo evidence of activated macrophages in human osteoarthritis. Osteoarthritis Cartilage 24: 1613-1621, 2016.

8. Sun H, Zhang Y, Song W, Yin L, Wang G, Yu D, Zhang Q, Yan X and $\mathrm{Li} \mathrm{S}$ : $\mathrm{IgM}^{+} \mathrm{CD} 27^{+} \mathrm{B}$ cells possessed regulatory function and represented the main source of B cell-derived IL-10 in the synovial fluid of osteoarthritis patients. Hum Immunol 80: 263-269, 2019.

9. Arkestål K, Mints M, Enocson A, Linton L, Marits P, Glise H, Andersson J and Winqvist O: CCR2 upregulated on peripheral T cells in osteoarthritis but not in bone marrow. Scand J Immunol 88: e12722, 2018.

10. Deligne C,CasulliS,Pigenet A,Bougault C,Campillo-Gimenez L, Nourissat G, Berenbaum F, Elbim C and Houard X: Differential expression of interleukin-17 and interleukin-22 in inflamed and non-inflamed synovium from osteoarthritis patients. Osteoarthritis Cartilage 23: 1843-1852, 2015.

11. de Munter W, Geven EJ, Blom AB, Walgreen B, Helsen MM, Joosten LA, Roth J, Vogl T, van de Loo FA, Koenders MI, et al: Synovial macrophages promote TGF- $\beta$ signaling and protect against influx of S100A8/S100A9-producing cells after intra-articular injections of oxidized low-density lipoproteins. Osteoarthritis Cartilage 25: 118-127, 2017.

12. Bondeson J, Wainwright SD, Lauder S, Amos N and Hughes CE: The role of synovial macrophages and macrophage-produced cytokines in driving aggrecanases, matrix metalloproteinases, and other destructive and inflammatory responses in osteoarthritis. Arthritis Res Ther 8: R187, 2006.

13. Barros MH, Hauck F, Dreyer JH, Kempkes B and Niedobitek G: Macrophage polarisation: An immunohistochemical approach for identifying M1 and M2 macrophages. PLoS One 8: e80908, 2013.

14. Varol C, Mildner A and Jung S: Macrophages: Development and tissue specialization. Annu Rev Immunol 33: 643-675, 2015.

15. Bi D, Zhou R, Cai N, Lai Q, Han Q, Peng Y, Jiang Z, Tang Z, Lu J, Bao W, et al: Alginate enhances Toll-like receptor 4-mediated phagocytosis by murine RAW264.7 macrophages. Int J Biol Macromol 105: 1446-1454, 2017.

16. Sanjuan MA, Dillon CP, Tait SW, Moshiach S, Dorsey F, Connell S, Komatsu M, Tanaka K, Cleveland JL, Withoff S, et al: Toll-like receptor signalling in macrophages links the autophagy pathway to phagocytosis. Nature 450: 1253-1257, 2007.

17. Hallowell RW, Collins SL, Craig JM, Zhang Y, Oh M, Illei PB, Chan-Li Y, Vigeland CL, Mitzner W, Scott AL, et al: mTORC2 signalling regulates $\mathrm{M} 2$ macrophage differentiation in response to helminth infection and adaptive thermogenesis. Nat Commun 8: 14208, 2017.

18. Qing L, Fu J, Wu P, Zhou Z, Yu F and Tang J: Metformin induces the M2 macrophage polarization to accelerate the wound healing via regulating $\mathrm{AMPK} / \mathrm{mTOR} / \mathrm{NLRP} 3$ inflammasome singling pathway. Am J Transl Res 11: 655-668, 2019.

19. Daghestani HN, Pieper CF and Kraus VB: Soluble macrophage biomarkers indicate inflammatory phenotypes in patients with knee osteoarthritis. Arthritis Rheumatol 67: 956-965, 2015.

20. Benito MJ, Veale DJ, FitzGerald O, van den Berg WB and Bresnihan B: Synovial tissue inflammation in early and late osteoarthritis. Ann Rheum Dis 64: 1263-1267, 2005.

21. Dragomir MP, Knutsen E and Calin GA: SnapShot: Unconventional miRNA functions. Cell 174: 1038-1038.e1, 2018.

22. D'Adamo S, Alvarez-Garcia O, Muramatsu Y, Flamigni F and Lotz MK: MicroRNA-155 suppresses autophagy in chondrocytes by modulating expression of autophagy proteins. Osteoarthritis Cartilage 24: 1082-1091, 2016. 
23. Rupaimoole R and Slack FJ: MicroRNA therapeutics: Towards a new era for the management of cancer and other diseases. Nat Rev Drug Discov 16: 203-222, 2017.

24. Yu J,Li Y,Pan Y,Liu Y, Xing H, Xie X, Wan D and Jiang Z: Deficient regulatory innate lymphoid cells and differential expression of miRNAs in acute myeloid leukemia quantified by next generation sequence. Cancer Manag Res 11: 10969-10982, 2019.

25. Soyocak A, Kurt H, Ozgen M, Turgut Cosan D, Colak E and Gunes HV: miRNA-146a, miRNA-155 and JNK expression levels in peripheral blood mononuclear cells according to grade of knee osteoarthritis. Gene 627: 207-211, 2017.

26. Zhang Y, Zhang M, Zhong M, Suo Q and Lv K: Expression profiles of miRNAs in polarized macrophages. Int J Mol Med 31: 797-802, 2013

27. Ma C, Wang Y, Shen A and Cai W: Resveratrol upregulates SOCS1 production by lipopolysaccharide-stimulated RAW264.7 macrophages by inhibiting miR-155. Int J Mol Med 39: 231-237, 2017.

28. Xu F, Kang Y, Zhang H, Piao Z, Yin H, Diao R, Xia J and Shi L: Akt1-mediated regulation of macrophage polarization in a murine model of Staphylococcus aureus pulmonary infection. J Infect Dis 208: 528-538, 2013.
29. Yang Y, Yang L, Liang X and Zhu G: MicroRNA-155 promotes atherosclerosis inflammation via targeting SOCS1. Cell Physiol Biochem 36: 1371-1381, 2015.

30. Cai X, Yin Y, Li N, Zhu D, Zhang J, Zhang CY and Zen K: Re-polarization of tumor-associated macrophages to pro-inflammatory M1 macrophages by microRNA-155. J Mol Cell Biol 4: 341-343, 2012.

31. Livak KJ and Schmittgen TD: Analysis of relative gene expression data using real-time quantitative PCR and the 2(-Delta Delta C(T)) method. Methods 25: 402-408, 2001.

32. Yu XM, Meng HY, Yuan XL, Wang Y, Guo QY, Peng J, Wang AY and Lu SB: MicroRNAs' involvement in osteoarthritis and the prospects for treatments. Evid Based Complement Alternat Med 2015: 236179, 2015.

33. Lu L, McCurdy S, Huang S, Zhu X, Peplowska K, Tiirikainen M, Boisvert WA and Garmire LX: Time series miRNA-mRNA integrated analysis reveals critical miRNAs and targets in macrophage polarization. Sci Rep 6: 37446, 2016.

(i) () () This work is licensed under a Creative Commons

EY Attribution-NonCommercial-NoDerivatives 4.0 International (CC BY-NC-ND 4.0) License. 